\title{
Inequalities in education and national income are associated with poorer diet in Europe: pooled analysis across 12 countries
}

\author{
H. L. Rippin 1 , J. Hutchinson ${ }^{1}$, D. C. Greenwood ${ }^{2}$, J. Jewell ${ }^{3}$, J.J. Breda ${ }^{3}$, A. Martin 4 , D. M. Rippin ${ }^{5}$,
} K. Schindler ${ }^{6}$, P. Rust ${ }^{6}$, S. Fagt ${ }^{7}$, J. Matthiessen ${ }^{7}$, E. Nurk ${ }^{8,9}$, K. Nelis ${ }^{8}$, M. Kukk ${ }^{8}$, H. Tapanainen ${ }^{10}$, L. Valsta ${ }^{10}$, T. Heuer ${ }^{11}$, E. Sarkadi-Nagy ${ }^{12}$, M. Bakacs ${ }^{12}$, S. Tazhibayev ${ }^{13}$, T. Sharmanov ${ }^{13}$, I. Spiroski ${ }^{14}$, M. Beukers ${ }^{15}$, C. van Rossum ${ }^{15}$, M. Ocke ${ }^{15}$, A. K. Lindroos ${ }^{16}$, Eva Warensjö Lemming ${ }^{16}$ and J. E. Cade $^{1}$ ${ }^{1}$ Nutritional Epidemiology Group (NEG), School of Food Science and Nutrition, University of Leeds, Leeds, LS2 9JT, ${ }^{2}$ Clinical and Population Science Department, Institute of Cardiovascular and Metabolic Medicine (LICAMM), University of Leeds, Leeds, LS2 9JT,

${ }^{3}$ Division of Noncommunicable Diseases and Promoting Health through the Life-Course, World Health Organization Regional Office for Europe, UN City, Marmorvej 51, 21000 Copenhagen, Denmark,

${ }^{4}$ Academic Unit of Health Economics, Leeds Institute of Health Sciences, University of Leeds, Leeds, LS2 9JT,

${ }^{5}$ Department of Environment and Geography, University of York, Wentworth Way, Heslington, York, YO10 5NG,

${ }^{6}$ Department of Nutritional Sciences, University of Vienna, Althanstrasse 14, 1090 Vienna, Austria,

${ }^{7}$ National Food Institute, Kemitorvet, Bygning 202, DK-2800 Kgs Lyngby, Denmark,

${ }^{8}$ Department of Nutrition Research, National Institute for Health Development, Tallinn, Estonia,

${ }^{9}$ Department of Nutrition, Institute of Basic Medical Sciences, University of Oslo, Norway,

${ }^{10}$ Public Health Promotion Unit, Finnish Institute for Health and Welfare, P.O. Box 30, FI-00271 Helsinki, Finland,

${ }^{11}$ Department of Nutritional Behaviour, Max Rubner-Institut, Federal Research Institute of Nutrition and Food, Karlsruhe, Germany,

${ }^{12}$ National Institute of Pharmacy and Nutrition; Budapest 1097 Hungary Albert Flórián út 3/A,

${ }^{13}$ Kazakh Academy of Nutrition, 66 Klochkov Street, Almaty 0500008, Republic of Kazakhstan,

${ }^{14}$ Institute of Public Health, 50. Divizija 6, 1000 Skopje, North Macedonia,

${ }^{15}$ National Institute for Public Health and the Environment, PO Box 1, 3720 BA Bilthoven, the Netherlands and

${ }^{16}$ Livsmedelsverket Swedish National Food Agency, Box 662, SE 75126 Uppsala, Sweden

Malnutrition linked to noncommunicable diseases (NCDs) presents major health problems across Europe. This includes overweight, obesity, cardiovascular disease (CVD) ${ }^{(1)}$ and micronutrient deficiency ${ }^{(2)}$. The World Health Organisation encourages countries to conduct national dietary surveys to obtain data to inform public health policies designed to prevent NCDs ${ }^{(3)}$. WHO recommended nutrient intakes (RNIs) of both macro and micronutrients are not widely achieved and few WHO European Member States report intakes by socioeconomic group ${ }^{(2)}$. This research therefore harmonises national individual-level dietary survey data from across the WHO European Region, exploring geographical variations in key nutrient intakes. It investigates between and within-country socioeconomic inequalities through measures of country-level Gross Domestic Product (GDP) and individual-level education.

Data on 27334 participants aged 19-64y were harmonised and pooled across nationally representative dietary survey datasets from 12 countries across the WHO European Region. Weighted mean nutrient intakes were age-standardised using the Eurostat 2013 European Standard Population ${ }^{(4)}$. Associations between country-level GDP and key nutrients and nutrient densities were investigated using linear regression. The potential mitigating influence of individual-level educational status was explored.

Higher GDP was positively associated with total sugar intake (5.0\% energy for each 10\% increase in GDP, 95\% CI $0.6 \%, 9.3 \%)$. Scandinavian countries had the highest vitamin D intakes and Central and Eastern European countries generally had lower total folate intakes. Participants with higher educational status had better nutritional intakes, particularly in lower GDP countries. In higher educated individuals a 10\% higher GDP was associated with lower total fat intakes $(-0.2 \%$ energy, $95 \%$ CI $-0.3 \%,-0.1 \%)$ and higher daily total folate intakes $(14 \mu \mathrm{g}, 95 \%$ CI $12 \mu \mathrm{g}, 16 \mu \mathrm{g})$.

Lower income countries and lower education groups had poorer diet, particularly for micronutrients. We demonstrate for the first time that higher educational status appears to mitigate the effects of poorer diets in lower income countries. Most countries had high energy and macronutrient intakes above the WHO RNIs; lower GDP countries may therefore follow the trajectory of higher income countries and face future elevated levels of obesity-related NCDs. This research reveals individual-level socioeconomic inequalities across WHO Europe, as those with less education generally had lower intakes of nutrients encouraged as part of a healthy diet, particularly iron and total folate. This work illustrates the feasibility and value of harmonising national dietary survey data to inform European policy regarding access to healthy diets, particularly in disadvantaged groups. It specifically highlights the need for strong policies supporting nutritional intakes, prioritising lower education groups and lower income countries to address the public health implications of the effect of GDP and education on nutritional intakes. Increasing educational levels will lead to better nourished populations, and the ability to improve GDP. Policies should therefore be put in place to achieve this.

\section{References}

1. World Health Organisation (2017). Geneva: WHO; [24/05/18]. http://www.who.int/en/news-room/fact-sheets/detail/obesity-and-overweight.

2. Rippin HL, Hutchinson J, Jewell J, et al.. (2017) Nutrients, 9(12):1288.

3. World Health Organisation (2014). European food and nutrition action plan 2015-2020. WHO Regional Office for Europe.

4. Eurostat (2013). Revision of the European Standard Population: report of Eurostat's taskforce. Luxembourg. 